\title{
Automated Anesthesia Delivery Pump
}

\author{
Hiranya Kudva ${ }^{1}$, Prof. Jyothi Warrier ${ }^{2}$ \\ ${ }^{1}$ (Biomedical, MGMCET/ Mumbai University, India) \\ 2 (Biomedical, MGMCET/ Mumbai University, India)
}

\begin{abstract}
The objective of the work has been to develop a system which independently controls the intravenous infusion rates of the hypnotic drug propofol and analgesic drug remifentanil. The system is designed to maintain a reference hypnotic depth level, while maintaining adequate analgesia. The main goals of general anesthesia are adequate hypnosis, analgesia and maintenance of vital functions. Furthermore the patient safety and cost reduction as a case of minimized drug consumption and shortened post- operative recovery phases are part of the main issues to motivate automation in anesthesia. Recent advances in sensing devices, along with robust nonlinear control theories, have generated new hopes that the gap between manual and automated control of anesthesia can finally be bridged.
\end{abstract}

Keywords: Analgesia, automated anesthesia, hypnosis, propofol, remifentanil, syringe pump.

\section{Introduction}

Anesthesia is defined as the absence or loss of sensation. In order to provide safe and adequate anesthesia, the anesthesiologist must guarantee analgesia, provide hypnosis, muscle relaxation and maintain vital functions of the patient. To assist access to internal organs and to depress movement response to surgical stimulation, muscle relaxation is necessary. Hypnosis, referred to as depth of anesthesia, is a general term indicating the unconsciousness and absence of post-operative recall of events that occurred during surgery. The electroencephalogram, which is the only non-invasive measure of central nervous system activity while the patient is unconscious, is considered as the major source of information to assess the level of hypnosis [1]. Analgesia is linked with pain relief. The electrocardiogram and response to nerve muscle stimulation is related to the extent of pain relief in the patient. An effort has been made to develop a closed-loop system that will automatically deliver hypnotic and analgesic drugs to the patient based on the EEG, ECG and galvanic skin response.

\section{Clinical significance}

Anesthesia is a state of the central nervous system in which the responses to noxious stimuli are suppressed reversibly. Few surgical procedures could be carried out without general anesthesia. Anesthesia can be produced by the ingestion of substances; by subcutaneous, intramuscular, intraperitoneal, and intravenous injection; by rectal administration; and by the inhalation of anesthetic gases or vapours. In all cases the agent must reach a critical concentration in the bloodstream to produce anesthesia [2].

\subsection{Functional Components Of Anesthesia}

Anesthesia can be conveniently classified into three functional components, each associated with a corresponding class of drugs. These functional components are shown in fig.1.

Hypnosis The main functional component of anesthesia is hypnosis, or the rendering of drug-induced unconsciousness. Its purpose is to take the patient to a state which inhibits the perception and recall of noxious stimuli.

Analgesia Although an adequate hypnotic state prevents the patient from perceiving or recalling noxious stimuli, their presence can still affect the hemodynamic state of the patient, respiration and hormonal secretion. This can be prevented by analgesic drugs which compromise the sympathetic and parasympathetic nervous systems.

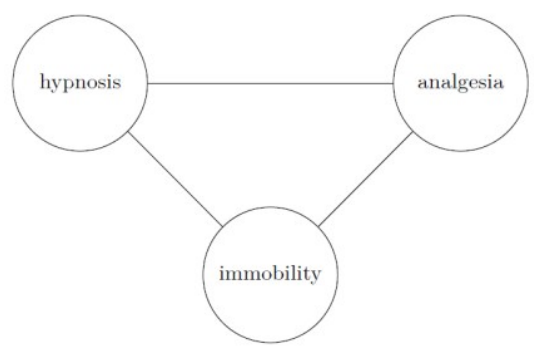

Fig.1. Functional Components of Anesthesia [3] 
Immobility Certain muscles, particularly abdominal ones, exhibit reflex activity. This activity is typically not blocked in an adequate hypnotic and analgesic state, which motivates the use of paralyzing drugs, establishing a neuromuscular blockade, during some surgical procedures. The resulting immobility component of anesthesia is completely decoupled from hypnosis and analgesia, which allows separate treatment from a control perspective.

\subsection{Anesthetic Drugs}

This section discusses some aspects of drugs used to provide an adequate hypnotic and analgesic state during surgical procedures.

Hypnotic Drugs - Propofol A drug of which the main purpose is to control the hypnotic state of the patient is referred to as a hypnotic drug. There exist a multitude of known hypnotic drugs, several of which are no longer used in clinical practice. Hypnotic drugs can be classified by means of administration; inhalation or intravenous infusion. The routinely used inhaled volatile drugs (nitrous oxide, isoflurane, desflurane and sevoflurane) are not purely hypnotic, but also provide analgesia to some extent. Routinely used intravenous drugs (propofol, ketamine, etomidate, midazolam, diazepam, lorazepam and thiopental) are pure hypnotic drugs, with the exception of ketamine which also provides analgesia to some extent. Propofol is a relatively new addition to this group, introduced in the 1990s. It has both fast redistribution and metabolism and does not accumulate in tissues unlike some of the other drugs. Particularly due to these properties, propofol has become widely accepted and the standard drug of choice for intravenous anesthesia.. Unlike inhaled drugs, it is not possible to measure the effect site (brain) concentration of propofol in real-time. This disadvantage is outweighed by the fast redistribution and metabolism of the drug, its lack of accumulation and the possibility of precise titration by means of electronically controlled intravenous infusion pumps. Besides, it is common practice to predict the effect site drug concentration using mathematical patient models. A major advantage of propofol, as compared to inhaled drugs, is that it is a pure hypnotic. This allows for decoupled control of the hypnotic and analgesic components of analgesia. It should be mentioned that this decoupling is, however, somewhat complicated by a synergistic interaction between propofol and several analgesic drugs. Another reason for the increasing popularity of propofol is that its use is associated with low incidence of postoperative nausea and vomiting compared to other hypnotic drugs. This decreases the duration of postoperative care and ultimately patient mortality. Furthermore, propofol lacks the strong hypotensive action common to inhaled drugs.

Analgesic Drugs - Remifentanil The fact that inhaled hypnotic drugs have analgesic properties enable their stand-alone use during surgical procedures involving limited noxious stimulation. During more stimulating procedures, or whenever the hypnotic drug of choice does not possess analgesic effect, an analgesic drug is coadministered with the hypnotic one. The most frequently used analgesic drugs are from the family of opioids (remifentanil, sufentanil, alfentanil, fentanyl, morphine and hydromorphine). In doses representative for clinical anesthesia, opioids do not exhibit hypnotic properties. However, there exists a synergistic effect between opioids and several of the hypnotic drugs, including propofol. This synergy enforces both the effect of the hypnotic and analgesic drug and plays a key role when the drugs are co-administered. Remifentanil is the most recent addition to the opioid family. It is characterized by its very fast redistribution and metabolism; remifentanil is the fastestacting of the opioids and its dynamics are of an order of magnitude faster than those of propofol. This makes remifentanil well suited for closed-loop controlled anesthesia and at the same time somewhat harder to manually titrate than the other opioids. The explanation of its fast metabolism is that remifentanil is metabolized by esterases in the blood plasma, while the metabolism of other opioids rely on hepatic biotransformation and renal excretion. This motivates the use of remifentanil in patients with liver or kidney conditions.

\section{Block Diagram}

The use of closed-loop systems in anesthesia can improve the quality of drug delivery. Closed-loop systems consist of a 'brain', a central operating system with built-in algorithms, an 'effect', a target control variable and an 'actuator', a drug delivery system, such as a syringe pump. These three elements are connected by a feedback system, which allows the automated control of drug delivery in order to maintain a pre-set target value of the control variable without any manual input. By frequent sampling of the control variable and more frequent changes of drug delivery rates than with manually delivered anesthesia, greater stability of the control variable may be achievable. The performance of a closed-loop system for anesthesia depends on the reliability of the control variable; therefore, adequate target parameters must be used for hypnosis and analgesia [4]. Automated anesthesia delivery implies that some form of electronic and/or mechanical instrumentation performs dose rate adjustments independent of human intervention. The desired target is set according to the feedback from the patient. Figure 2 shows the block diagram for the automated anesthesia delivery pump. 


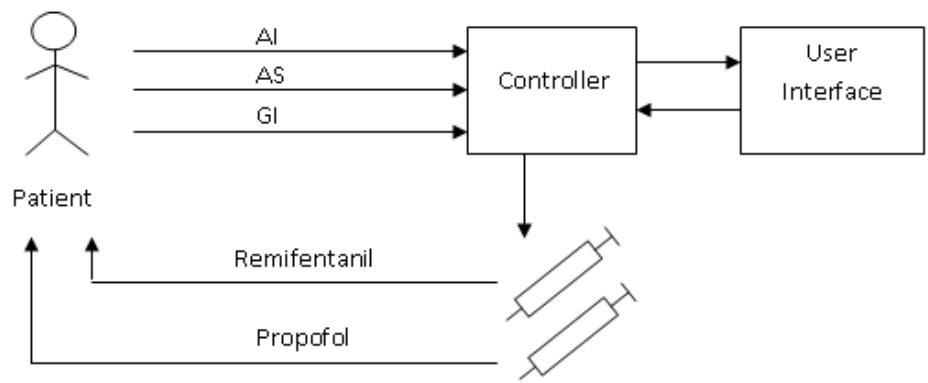

Fig.2. Block Diagram

Outputs from Patient The patient is connected various electrodes to measure EEG, ECG and GSR. The signals acquired by the patient are processed to get desired values which will be utilized by the controller to set appropriate anesthesia doses. The outputs are continuously measured during anesthesia delivery to maintain adequate hypnosis and analgesia. This acts as a feedback to the controller. Anesthesia Index (AI) is a parameter derived from EEG which is used to measure the depth of hypnosis. Depth of analgesia is determined by AnalgoScore (AS), which is derived from ECG, and GSR Index (GI). The Anesthesia Index algorithm derives the AI by wavelet decomposition of the EEG signal. The anesthesia Index (AI) is a dimensionless number derived from processing the component frequencies of the EEG. It ranges from 0 (isoelectric brain activity) to 100 (consciousness). A value from 40 to 60 is considered as representing an adequate state of hypnosis. The AnalgoScore (AS) is derived from the ECG. This is an index which ranges from -9 to +9 . A value between -3 and +3 represents adequate analgesia. The Galvanic Skin Response (GSR) also indicates the extent of pain or pain relief of the patient. The GI is a value from 0 to 5 where 0 represents extreme pain and 5 represents no pain. Controller The various inputs are given to the controller which calculates the appropriate doses of anesthetic drugs to be given to the patient. The controller can range from a simple PID controller to a more complex fuzzy controller [5]. The controller initiates the actuator system which will administer required propofol dose based on the AI. The analgesic drug remifentanil is administered based on the AS and GI.

Actuator system Here the actuator is a drug delivery system. It consists of infusion devices to accurately regulate the amount of intravenous drugs administered to the patient. There are two types of powered infusion devices. The oldest established is the syringe pump in which the barrel of the syringe held firm in the device and the mechanism pushes the syringe plunger at a predetermined rate, so delivering fluid into the patients. The alternative mechanism for powered infusion devices is peristaltic pump device which essentially involves a rhythmic squeezing of the infusion set tubing, proximal to distal, thus displacing fluid along the tube from bag or bottle to patient.

User interface As in any other system, user interface is needed in this system for better control of anesthesia delivery. The dose of anesthesia drugs depends on age and weight of the patient. This information has to be fed by the anesthesiologist. Also at times of failure of controller or increased noise in the signal, the anesthesiologist takes over the machine to administer drugs manually.

\subsection{The Syringe Pump}

\section{Design And Implementation}

The drug delivery system used in this project is a syringe pump which consists of two syringe assemblies to deliver drugs to the patient. The syringe pump is illustrated in fig.3. The plunger of the syringe is controlled by a dc motor. A multi-turn potentiometer is attached to the shaft of the motor. The potentiometer acts as a shaft encoder to determine the angle made by the motor shaft and thus the position of the plunger. Forward motion of the plunger will expel fluid and reverse motion will withdraw fluid into the syringe. The syringe pump is designed to continuously fill and expel drug. There are two solenoid valves placed at the inlet and outlet of the syringe to check the flow of drug. While filling the syringe, the valve 1 will be kept open and valve 2 will be kept closed. This will allow the drug to go into the syringe instead of going to the patient. While administering drug to the patient, valve 1 is kept close and valve 2 is opened. This will cause the fluid in the syringe to be delivered to the patient instead of back flow into the reservoir. 


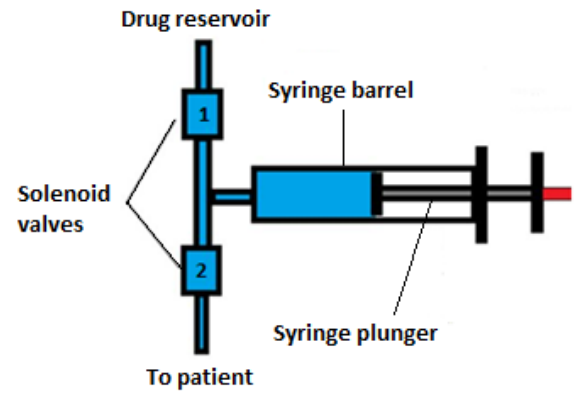

Fig.3. Illustration of the Syringe Pump System

\subsection{Microcontroller}

The infusion devices are controlled by the microcontroller. The microcontroller will be programmed to control the operation of the delivery system. After selecting a suitable dose and period, the microcontroller will give signals that drive the motor and the valves and consequently driving the syringe pump to deliver the required dose. The controller also displays the inputs from the patient continuously and the amount of drug administered to the patient. PIC 16F877A is one of the most advanced microcontroller from Microchip. This controller is widely used for experimental and modern applications because of its low price, wide range of applications, high quality and ease of availability. It is ideal for applications such as machine control applications and measurement devices. The following figure shows the pin configuration of PIC16F877A.

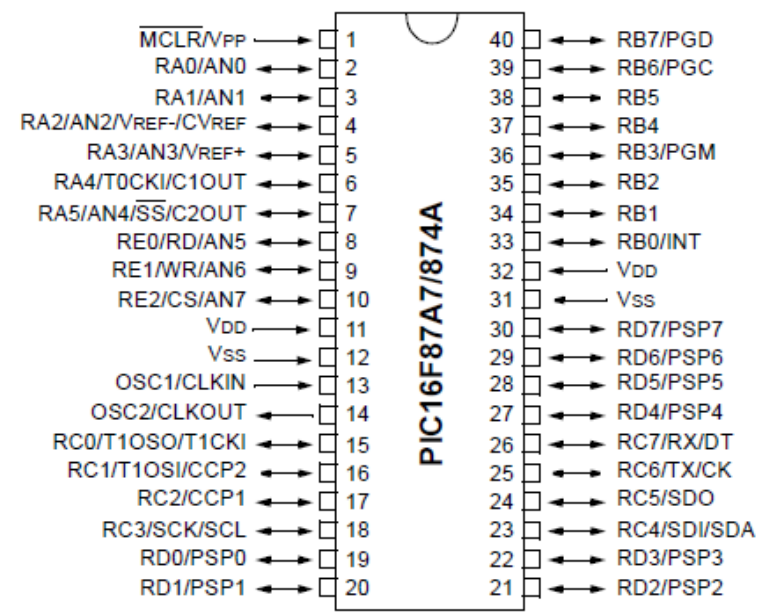

Fig.4. Pin diagram of PIC16F877A

\subsection{Motor Driver}

Most of the DC motors have power requirements well out of the reach of a microcontroller and more over the voltage spikes produced while reversing the direction of rotation could easily damage the microcontroller. So it is not wise to connect a DC motor directly to the microcontroller. The perfect solution is to use a motor driver circuit in between the microcontroller and the DC motor. We can easily control the switching of L293D using a microcontroller.

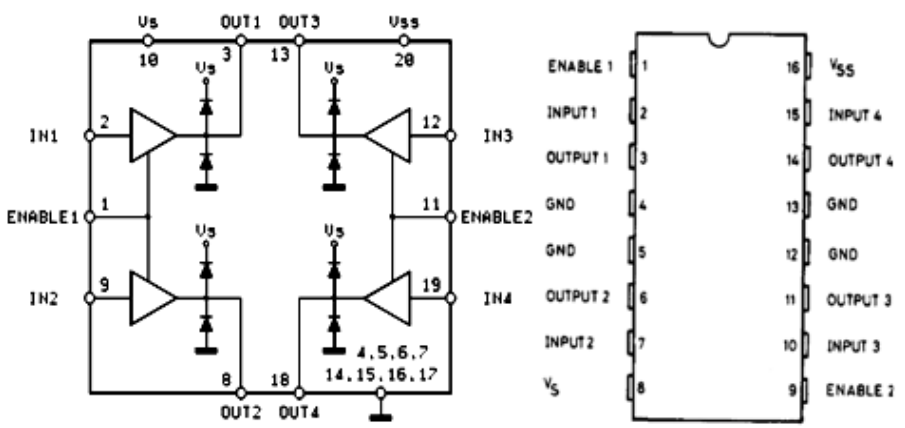

Fig.5. Block Diagram and Pin Diagram of L293D 
L239D can provide a maximum current of $600 \mathrm{~mA}$ from $4.5 \mathrm{~V}$ to $36 \mathrm{~V}$. All inputs of this IC are TTL compatible and clamp diodes are provided with all outputs. They are used with inductive loads such as relays solenoids, motors etc. They are fitted with internal high speed clamp diodes for inductive spike protection. Other good features are high noise immunity, internal ESD protection, thermal shutdown, separate input supply for each channel etc. We can drive two motors or four solenoid valves simultaneously with one IC. Fig.5 shows the block diagram and pin diagram of L293D.

A simple schematic for interfacing two DC motors using L293D is shown in fig.6. Three Pins are needed for interfacing a DC motor (A, B, Enable). Output is to be enabled completely therefore it can connect be connected to VCC and only two pins needed from controller to make the motor work. Table 1 shows the truth table (one channel) of L293D.

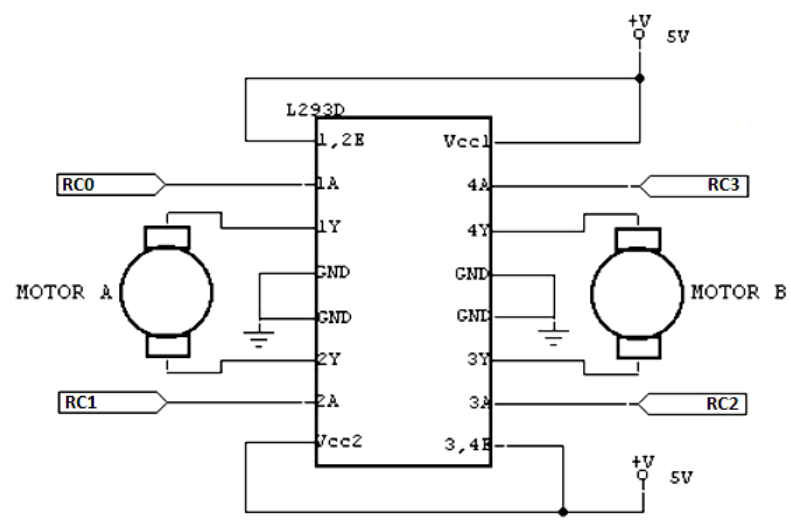

Fig.6. Interfacing of DC motors with L293D

Table 1 Truth Table of L293D

\begin{tabular}{|c|c|c|c|}
\hline Input (A) & Enable $(*)$ & Output(Y) & Description \\
\hline H & $\mathrm{H}$ & $\mathrm{H}$ & Motor stops \\
\hline $\mathrm{L}$ & $\mathrm{H}$ & $\mathrm{L}$ & Motor runs anticlockwise \\
\hline $\mathrm{H}$ & $\mathrm{L}$ & $\mathrm{Z}$ & Motor runs clockwise \\
\hline $\mathrm{L}$ & $\mathrm{L}$ & $\mathrm{Z}$ & Motor stops \\
\hline
\end{tabular}

$\mathrm{Z}=$ High output impedance

(*) Relative to the considered channel

Similarly, the driver can be used to drive a solenoid valve instead of DC motor. The solenoid valve is operated in one direction only. Hence four solenoid valves can be connected at the four output pins of L293D and interfaced with the microcontroller.

\subsection{Interfacing Of Microcontroller}

The outputs from the patient (AI, AS and GI) are to be given to the microcontroller to calculate the drug dose. In absence of a real patient, the outputs are taken from three potentiometers which can be varied to get different values of AI, AS and GI respectively. The outputs are analog in nature and are given directly to the controller via the analog input ports. These parameters decide whether the drug should be infused or not based on the flowchart shown below. 


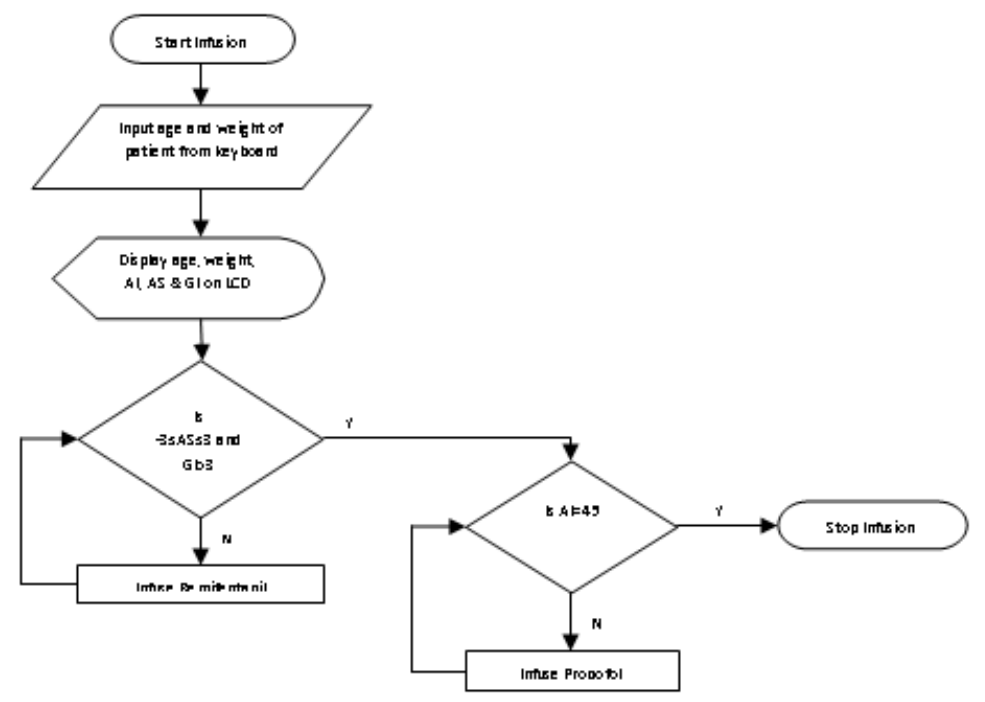

To infuse the drug, the microcontroller gives command to the specific motor and solenoid valves based on the position of the syringe plunger which is decided by the voltage on the respective multi-turn potentiometer. The output of this potentiometer is also analog and is given to the controller via the analog ports. The controller is interfaced with a 4x3 keypad for entering age and weight of the patient. A $16 \times 2$ LCD is connected in 4 bit mode to reduce the number of pin connections. The LCD displays the age and the weight entered by the user. It refreshes to continuously display the AI, AS and GI parameters of the patient. After infusion of each drug, it displays the amount of drug delivered. The following figure shows the interfacing of each component with the microcontroller.

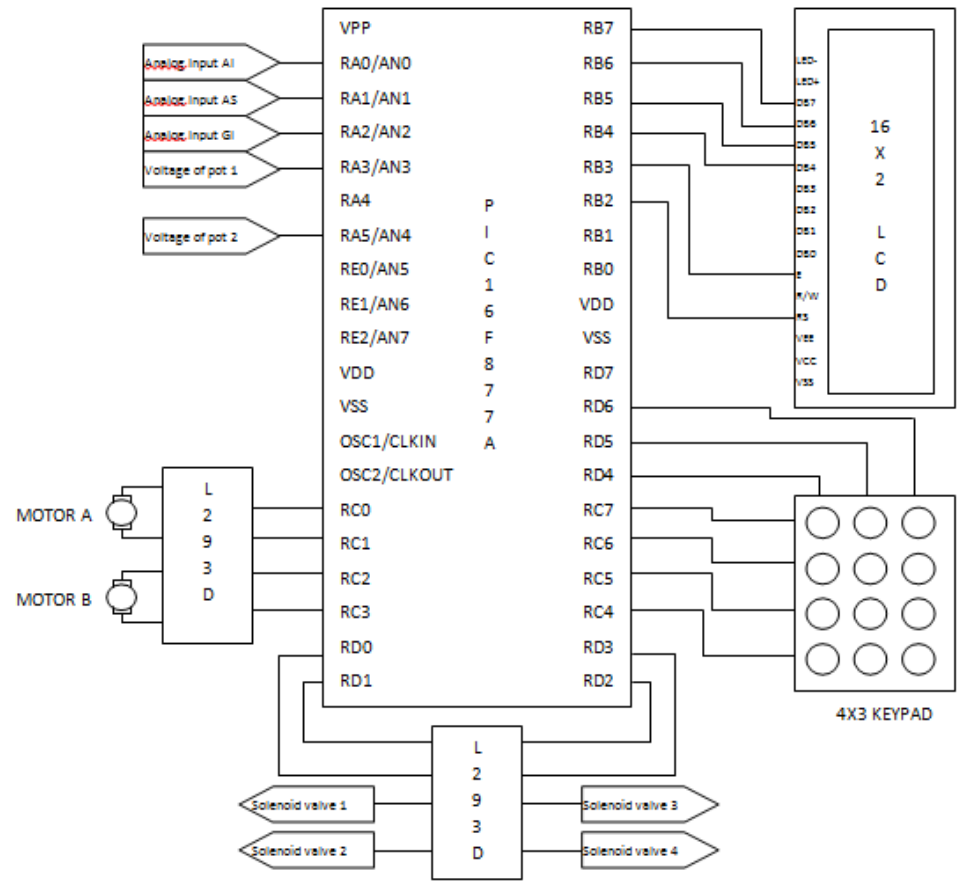

Fig.7. Interfacing of Microcontroller

\subsection{Drug Dose Calculation}

The microcontroller calculates the amount of each drug to be administered based on the age and weight of the patient. The doses differ for paediatric and adult patients. For an adult, remifentanil is infused for $3 \mathrm{~min}$, with a dose of $0.5 \mathrm{mcg} / \mathrm{kg} / \mathrm{min}$ for the first 2 minutes and with a dose of $0.2 \mathrm{mcg} / \mathrm{kg} / \mathrm{min}$ for the third minute. Remifentanil is then injected at a dose of $0.1 \mathrm{mcg} / \mathrm{kg} / \mathrm{min}$ for the remainder of the induction phase. Propofol induction starts $2 \mathrm{~min}$ after the beginning of the remifentanil infusion with a dose varying from 1.5 to $2 \mathrm{mg} / \mathrm{kg}$ 
[4]. The dose during the induction phase is to be injected by the anaesthesiologist. The system here is designed for the maintenance of anesthesia during the surgery. During the maintenance period, remifentanil is infused at a rate of $0.1 \mathrm{mcg} / \mathrm{kg} / \mathrm{min}$ and propofol is infused at a rate of $0.5 \mathrm{mg} / \mathrm{kg} / \mathrm{min}$.

The dose is calculated using the following equation,

Volume to be administered $=\frac{\text { Dose ordered }}{\text { Dose available }} \times$ Volume available

Using this equation, the volume of drug to be administered is calculated to be $0.002 \mathrm{ml} / \mathrm{kg} / \mathrm{min}$ for $50 \mathrm{mcg} / \mathrm{ml}$ solution of remifentanil and $0.05 \mathrm{ml} / \mathrm{kg} / \mathrm{min}$ for $10 \mathrm{mg} / \mathrm{ml}$ solution of propofol during the maintenance phase.

\section{Results And Discussions}

The automated anesthesia delivery pump designed here was tested for its accuracy. The amount of each drug varies according to the input given by the user. The system was tested by inputting different values of age and weight. Also, each time, the AI, AS and GI values were changed to observe the output of the system. The observations were as follows.

Table 2 Output of the system

\begin{tabular}{|c|c|c|c|c|c|c|c|c|c|}
\hline Sr.No. & Age & Weight $(\mathrm{Kg})$ & AI & AS & GI & \multicolumn{2}{|c|}{ Dose ordered (ml) } & \multicolumn{2}{c|}{ Dose delivered (ml) } \\
\cline { 7 - 11 } & & & & & & Remifentanil & Propofol & Remifentanil & Propofol \\
\hline 1 & 30 & 65 & 39 & +5 & 2.5 & 0.13 & 0.00 & 0.12 & 0.00 \\
\hline 2 & 30 & 65 & 52 & +4 & 2.9 & 0.13 & 3.25 & 0.12 & 3.20 \\
\hline 3 & 30 & 65 & 50 & +2 & 3.2 & 0.00 & 3.25 & 0.00 & 3.20 \\
\hline 4 & 55 & 85 & 70 & -6 & 2.8 & 0.17 & 4.25 & 0.16 & 4.20 \\
\hline 5 & 55 & 85 & 45 & -1 & 3.7 & 0.00 & 0.00 & 0.00 & 0.00 \\
\hline
\end{tabular}

The above table shows that the system can deliver drugs to the accuracy of $0.01 \mathrm{ml}$ in case of remifentanil and $0.1 \mathrm{ml}$ in case of propofol. It does not deliver the respective drug when the conditions for hypnosis or analgesia or both are met.

\section{Conclusion}

A system which will automatically monitor the anesthesia level of the patient and administer drugs when required has been developed here. This system will not eliminate the anesthesiologist but will allow him to better and more safely perform his or her job. Automation of anesthesia for monitoring of vital functions is desirable as it will provide more time and flexibility to the anesthesiologist to focus on critical issues, monitor the conditions that cannot be easily measured and overall improve patient's safety. Also, the cost of the drugs will be reduced and shorter time will be spent in the postoperative care unit. We will evaluate this proposed system and compare its performance against existing methods in order to claim the effectiveness and efficiency of this proposed work.

\section{Acknowledgements}

We would like to thank our head of the department Dr. G.D. Jindal for giving us the freedom to select our desired topic for the project work and supporting us in our endeavors.

\section{References}

[1] P. Dua and E. N. Pistikopoulos, "Modelling and control of drug delivery systems," Comps and Chem Eng, vol. $29,2005$.

[2] L. A. Geddes and L. E. Baker, "Anesthesia and Anesthesia Equipment,” in Principles of Applied Biomedical Instrumentation, 3rd ed. New Delhi, India: Wiley India, 2008, ch. 15, pp. 901-904.

[3] K. Soltész, "On automation in anesthesia", Ph.D. Thesis, Dept. Automat. Control, Lund Univ, Lund, Sweden, 2013.

[4] T. M. Hemmerling et al., "Evaluation of a novel closed-loop total intravenous anesthesia drug delivery system: a randomized controlled trial”, BJA, Feb. 2013.

[5] O. Simanski et al., "Automatic drug delivery in anesthesia: From the beginning until now," in $15^{\text {th }}$ Mediterranean Conference on Control and Automation, Athens - Greece, Jul. 27-29, 2007. 